\title{
Tumor Budding in Oral Cavity Cancer
}

\author{
Fernando Candanedo Gonzalez*, Orlando Emmanuel Falcon Antonio, Juan de Dios Bolaños De \\ la Torre and Armando Gamboa Dominguez
}

Department of Pathology, Instituto Nacional de Ciencias Medicas y Nutricion Salvador Zubiran, Mexico

*Corresponding author: Fernando Candanedo-Gonzalez, Department of Pathology, Instituto Nacional de Ciencias Medicas y Nutricion Salvador Zubiran, Mexico.

To Cite This Article: Fernando Candanedo-Gonzalez, Tumor Budding in Oral Cavity Cancer. 2020 - 7(3). AJBSR.MS.ID.001160.

DOI: 10.34297/AJBSR.2020.07.001160.

Received: 眥 January 29, 2020; Published: 䟧 February 19, 2020

\begin{abstract}
Tongue squamous cell carcinoma (TSCC) is the most common carcinoma in the oral cavity with a tendency to rapid invasion and local spread and high recurrence rate. The detection of TSCC at an early stage (T1/T2N0M0) does not always predict a good prognosis. Therefore, it is important to identify prognostic markers that can be evaluated in biopsies. Tumor budding (TB) histologically represent the epithelial mesenchymal transition (EMT) and specifically in TSCC, TB correlates with hidden lymph node metastases and poor prognosis. The objetive of this review is to summarize the evidence supporting the implementation of TB in the TSCC as a predictive biological marker of poor prognosis.
\end{abstract}

Keywords: Tumor budding, Oral cancer, Tongue squamous cell carcinoma, Prognosis

\section{Mini-Review}

In 1982, Elizabeth Dexter Hay was the first to describe the process of epithelial-mesenchymal transition (EMT), in vivo in chickens during embryogenesis [1]. He identified the mesenchymal transformation of epithelial cells by suspending them in collagen gels [2]. He also observed that epithelial cells lose their apical-basal polarity and union architecture to become mesenchymal cells with greater migratory capacity [3]. Later, it was observed that EMT is a central element not only in embryonic development, but also in the process of wound healing and in the migration of tumor cells. EMT is classified into 3 types according to the process involved: type 1 for gastrulation and embryogenesis, type 2 for regeneration and wound healing and type 3 for carcinogénesis [4].

By light microscopy, the histological representation with hematoxylin and eosin of EMT is defined by the presence of individual cells and in groups of up to four tumor cells separated from the invasion front, called tumor budding (TB) [5]. This biological phenomenon is a dynamic process carried out by a tumor with the potential to invade other tissues, spread and metastasize. In 1987, Hayashida et al. [6]. They were the first to study the phenomenon of TB in colorectal cancer (CRC). TB is a predictor of metastatic disease to distant lymph nodes, local recurrence, shorter overall survival time and disease-free survival and an independent prognostic factor. This allows stratification of patients in risk categories more significant than those defined by the TNM stage, and to guide treatment decisions, especially in CRC T1 and T3 N0 (Stage II, Dukes' B). It has been reported that TB is a promising prognostic marker in other types of cancer. Therefore, the biological implications of TB in CRC have been extrapolated to esophageal cancer [7,8], head and neck [9] and tongue squamous cell carcinoma (TSCC) [10].

In these sites the presence of TB has also been associated with poor prognosis. The objective of this review is to summarize the evidence supporting the implementation of TB in the TSCC as a predictive biological marker of poor prognosis. TSCC is the most common carcinoma of the oral cavity. According to the American Cancer Society, 17,060 new cases of tongue cancer are estimated each year [11], which represents $30 \%$ of all oral cavity cancers, with an increase in their incidence [12]. The TSCC is significantly more aggressive than other forms of oral cancer, with a tendency to rapid local invasion and spread and high recurrence rate [13-15].

The detection of TSCC at an early stage (T1/T2N0M0) does not always predict a good prognosis since $20 \%$ to $40 \%$ of cases already 
have hidden metastases in the presentation. Because the size (T1 or T2N0M0) of the TSCC alone has consistently failed to distinguish these 2 groups. On the other hand, the World Health Organization (WHO) graduation system for early stage TSCC has a low prognostic value. Therefore in 2011, [10], were the first to observe that the presence of TB in TSCC was associated with poor prognosis. In 2018, in a meta-analysis $[9,16]$, showed that TB is significantly associated with lymph node metastases, disease-free survival and overall survival [17], observed that the histopathological graduation of the WHO improved significantly with the incorporation of TB. Specifically in TSCC, TB correlates with hidden lymph node metastases and poor prognosis [18-20]. In addition, it is a simple, reliable and reproducible method in biopsies. Therefore, they suggest that it should be included in the pathology reports [21]. In conclusion, the evidence indicates that the presence of TB is a poor prognostic factor that can help stratify patients by risk groups.

\section{Conflict of Interest}

The authors declare no conflict of interest.

\section{References}

1. Kong D, Li Y, Wang Z, Sarkar FH (2011) Cancer stem cells and epithelialto-mesenchymal transition (EMT)-phenotypic cells: are they cousins or twins? Cancers (Basel) 3(1): 716-729.

2. Greenburg G, Hay ED (1982) Epithelia suspended in collagen gels can lose polarity and express characteristics of migrating mesenchymal cells. J Cell Biol 95(1): 333-339.

3. Guarino M, Rubino B, Ballabio G (2007) The role of epithelialmesenchymal transition in cancer pathology. Pathology 39(3): 305-318.

4. Prat A, Perou CM (2011) Deconstructing the molecular portraits of breast cancer. Mol Oncol 5(1): 5-23.

5. Lugli A, Kirsch R, Ajioka Y, Bosman F, Cathomas G et al. (2017) Recommendations for reporting tumor budding in colorectal cancer based on the International Tumor Budding Consensus Conference (ITBCC) 2016. Mod Pathol 30(9): 1299-1311.

6. Hayashida K, Isomoto $\mathrm{H}$, Shirouzu K (1987) A study of invasive colorectal carcinoma with reference mainly to vessel invasion and budding. Nippon Daicho-komonbyo Gakkai Zasshi 40: 119-126.

7. Roh MS, Lee JI, Choi PJ (2004) Tumor budding as a useful prognostic marker in esophageal squamous cell carcinoma. Dis Esophagus 17(4): 333-337.

8. Koike M, Kodera Y, Itoh Y, Nakayama G, Fujiwara M, et al. (2008) Multivariate analysis of the pathologic features of esophageal squamous cell cancer: Tumor budding is a significant independent prognostic factor. Ann Surg Oncol 15(7): 1977- 1982
9. Almangush A, Salo T, Hagström J, Leivo I (2014) Tumour budding in head and neck squamous cell carcinoma - a systematic review. Histopathology 65(5): 587-594.

10. Wang C, Huang H, Huang Z, Wang A, Chen X, et al. (2011) Tumor budding correlates with poor prognosis and epithelial-mesenchymal transition in tongue squamous cell carcinoma. J Oral Pathol Med. 40(7): 545-551.

11. Siegel R, Miller KD, Jemal A (2019) Cancer Statistics, 2019. CA Cancer J Clin 69(1): 7-34.

12. Ng JH, Iyer NG, Tan MH, Edgren G (2017) Changing epidemiology of oral squamous cell carcinoma of the tongue: A global study. Head Neck 39(2): 297-304.

13. Lydiatt DD, Robbins KT, Byers RM, Wolf PF (1993) Treatment of stage I and II oral tongue cancer. Head Neck 15(4): 308-312.

14. Yuen AP, Lam KY, Chan AC, Wei WI, Lam LK, et al. (1999) Clinicopathological analysis of elective neck dissection for N0 neck of early oral tongue carcinoma. Am J Surg 177(1):90-92.

15. Farhood Z, Simpson M, Ward GM, Walker RJ, Osazuwa-Peters N, et al. (2019) Does anatomic subsite influence oral cavity cancer mortality? A SEER database analysis. Laryngoscope 129(6): 1400-1406.

16. Almangush A, Pirinen M, Heikkinen I, Mäkitie AA, Salo T (2018) Tumour budding in oral squamous cell carcinoma: a meta-analysis. Br J Cancer 118(4): 577-586

17. Elseragy A, Salo T, Coletta RD, Kowalski LP, Haglund C, et al. (2919) A proposal to revise the histopathologic grading system of early oral tongue cancer incorpirating tumor budding. Am J Surg Pathol 43(5): 703-709.

18. Xie N, Wang C, Liu X, Li R, Hou J, et al. (2015) Tumor budding correlates with occult cervical lymph node metastases and poor prognosis in clinical early-stage tongue squeamous cell carcinoma. J Oral Pathol Med 44(4): 266-72.

19. Almangush A, Bello IO, Keski-Säntti H, Mäkinen LK, Kauppila JH, et al. (2014) Depth of invasión, tumor budding and worst pattern of invasión: prognostic indicators in early-stage oral tongue cancer. Head Neck 36(6): 811-818

20. Chatterjee D, Bansal V, Malik V, Bhagat R, Punia RS, et al. (2019) tumor budding and worse pattern of invasion can predict nodal metastasis in oral cancers and associated with poor survival in early-stage tumors. Ear Nose Throat J 98(7): E112-E119.

21. Xie N, Yu P, Liu H, Liu X, Hou J, et al. (2019) Validation of the International Tumor Budding Consensus Conference (2016) recommendations in oral tongue squamous cell carcinoma. J Oral Pathol Med 48(6): 451-458. 